\title{
Estudo do Campo Próximo de Nanoantenas Formadas por Partículas Esféricas de Ouro
}

\author{
Karlo Q. da Costa e Victor A. Dmitriev
}

\begin{abstract}
Resumo-Neste trabalho é apresentado um estudo do campo próximo de nanoantenas formadas por partículas de ouro. As partículas analisadas são do tipo esféricas, sendo que dois diferentes casos são considerados. $O$ primeiro com uma única esfera e o segundo com duas esferas. Por meio do modelo analítico de Mie e o Método dos Momentos (MoM), várias nanoantenas com diferentes dimensões foram investigadas na faixa de comprimentos de onda de $500 \mathrm{~nm}$ a $1000 \mathrm{~nm}$. Os resultados obtidos são analisados e comparados em termos de intensidade e localização do campo elétrico próximo.
\end{abstract}

Palavras-Chave-Nanoantenas, análise de campo próximo, espalhamento eletromagnético, Método dos Momentos (MoM).

Abstract-This work presents a near field analysis of nanoantennas composed by small gold particles. The particles analyzed are of spherical type, where two different cases are considered. The first is one single sphere and the second two spheres. By the analytical Mie scattering model and the Method of Moments (MoM), several nanoantennas with different sizes were investigated in the wavelength range of $500 \mathrm{~nm}$ to $1000 \mathrm{~nm}$. The results obtained are analyzed and compared in terms of intensity and localization of the electric near field.

Index Terms-Nanoantennas, near field analysis, electromagnetic scattering, Method of Moments (MoM).

\section{INTRODUÇÃO}

O problema de espalhamento de ondas eletromagnéticas por metais em freqüências ópticas possui características especiais. Nestas freqüências, os metais exibem oscilações elétricas chamadas de plasmons [1], as quais produzem campos eletromagnéticos intensos na superfície dos metais. As ressonâncias destas oscilações dependem das propriedades elétricas do metal, de suas dimensões e da direção e polarização da onda eletromagnética incidente. Estas ressonâncias podem ocorrer inclusive em estruturas metálicas com dimensões menores que o comprimento de onda de operação. O modelo da permissividade complexa de LorentzDrude pode ser utilizado como ponto de partida para estudar este fenômeno [2].

O estudo da interação da luz com nanoestruturas metálicas é chamado de plasmônica ou nanoplasmônica [1]-[2]. Este ramo tem recebido recentemente bastante atenção de pesquisadores devido as interessantes possibilidades futuras de aplicações. Alguns exemplos de promessas de aplicações

Karlo Q. da Costa e Victor A. Dmitriev, Faculdade de Engenharia Elétrica - ITEC, Universidade Federal do Pará, Belém/PA, Brasil, Emails: karlo@ufpa.br, victor@ufpa.br. Este trabalho foi realizado com apoio financeiro do CNPq. da plasmônica são microscópios ultra-sensíveis, chips de computador super rápidos, armazenamento de dados ópticos e elementos de circuitos funcionais de tamanhos nanométricos. Existem também promessas de aplicações na medicina para tratamento de Câncer [3].

Com o desenvolvimento da plasmônica, surgiu o conceito de nanoantenas, também conhecidas como antenas ópticas, que são estruturas metálicas extremamente pequenas, na ordem de nanômetros, que podem transmitir, receber, confinar e amplificar a luz [4]. A principal característica destas antenas é que elas são ressonantes em dimensões bem menores que o comprimento de onda da luz. Estas características são desejáveis para a manipulação e controle da luz em escalas menores que o comprimento de onda.

Em [5] é apresentado um exemplo de nanoantena aplicada para transmissão e recepção de luz. Neste artigo os autores acoplaram um guia de onda dielétrico a uma nanoantena formada por um cilindro dielétrico coberto por uma camada de ouro. Nesta configuração foi observado que o comprimento de onda de ressonância deste sistema pode ser controlado pelas espessuras das camadas de dielétrico e ouro da nanoantena cilíndrica. Nanoantenas utilizadas para aplicação em amplificação e confinamento do campo próximo podem ser encontradas em [6]-[13]. Em [6]-[8] são analisadas antenas do tipo barra cilíndrica e dipolos de diferentes tamanhos. São apresentados resultados teóricos e experimentais sobre o comportamento ressonante destas antenas. Um monopolo alimentado por uma abertura circular é apresentado em [9]. Antenas do tipo dipolos bowtie são investigadas em [10]-[11]. Esferas de ouro com diferentes dimensões e distâncias entre si são analisadas em [12]-[13].

No presente trabalho são analisadas nanoantenas formadas por esferas de ouro de diferentes dimensões e distâncias entre si na faixa de comprimentos de onda de 500nm a $1000 \mathrm{~nm}$. Dois casos distintos são analisados, um com uma única esfera, e o outro com duas esferas. O modelo analítico de espalhamento de Mie e o Método dos Momentos (MoM) são utilizados para os cálculos numéricos. Várias configurações diferentes de nanoantenas esféricas são analisadas e comparadas em termos de intensidade e confinamento do campo elétrico próximo.

\section{MODELAGEM TEÓRICA}

O problema de análise em questão é de espalhamento eletromagnético, onde uma onda plana incide sobre partículas esféricas de ouro. No caso de só uma esfera, foi utilizado o 
modelo analítico de Mie [14] e o método numérico MoM com a mesma modelagem apresentada em [15], a qual considera a equação integral tensorial do campo elétrico. Já para o caso de duas esferas foi utilizado apenas o MoM para os cálculos.

A permissividade complexa das esferas de ouro foi representada pelo modelo de Lorentz-Drude com um termo de interbanda. Esta permissividade é dada por $\varepsilon_{2}=\varepsilon_{0} \varepsilon_{\mathrm{r}}$, onde [2]

$$
\varepsilon_{r}=1-\frac{\omega_{p}^{2}}{\omega^{2}-j \Gamma \omega}+\frac{\tilde{\omega}_{p}^{2}}{\omega_{0}^{2}-\omega^{2}+j \gamma \omega}+6
$$

sendo $\omega_{p}=13,8 \times 10^{15} \mathrm{~s}^{-1}$ e $\Gamma=1,075 \times 10^{14} \mathrm{~s}^{-1}$ a frequência de plasma e de colisão dos elétrons livres, respectivamente. As características de ressonância do primeiro termo de interbanda dos elétrons ligados são $\omega_{0}=2 \pi c / \lambda_{0}, \lambda_{0}=450 \mathrm{~nm}$, $\tilde{\omega}_{p}=45 \times 10^{14} \mathrm{~s}^{-1}$ e $\gamma=9 \times 10^{14} \mathrm{~s}^{-1}$. Nesta expressão foi utilizado o fator $\exp (j \omega t), \omega$ a freqüência angular e $\varepsilon_{0}$ a permissividade do espaço livre. Este modelo é válido para $\lambda>500 \mathrm{~nm}$.

Baseado nestes modelos matemáticos, diversos códigos em matlab foram desenvolvidos para os cálculos numéricos. Nas próximas seções são apresentados os resultados destes cálculos e as respectivas análises.

\section{RESUlt AdOS PARA O CASO DE UMA ESFERA}

A geometria da nanoantena do primeiro caso analisado, com uma esfera, é apresentada na Fig. 1. Nesta figura uma esfera de ouro de raio $a$, inserida no ar, está centralizada na origem do sistema de referência. A onda plana que incide na esfera está polarizada em $x$ e se propaga para $+z$. A expressão matemática desta onda plana é

$\bar{E}_{i}=E_{i 0} e^{j(\omega t-k z)} \bar{a}_{x}$

$\bar{H}_{i}=\frac{E_{i 0}}{\eta} e^{j(\omega t-k z)} \bar{a}_{y}$

onde $E_{i 0}$ é a amplitude do campo elétrico incidente, $k=\omega\left(\mu_{0} \varepsilon_{0}\right)^{1 / 2}=2 \pi c / \lambda$ a constante de propagação no ar, $\lambda$ é o comprimento de onda, $c$ é a velocidade da luz no ar, $\mu_{0}$ a permeabilidade magnética do espaço livre e $\eta=120 \pi$ a impedância do espaço livre.

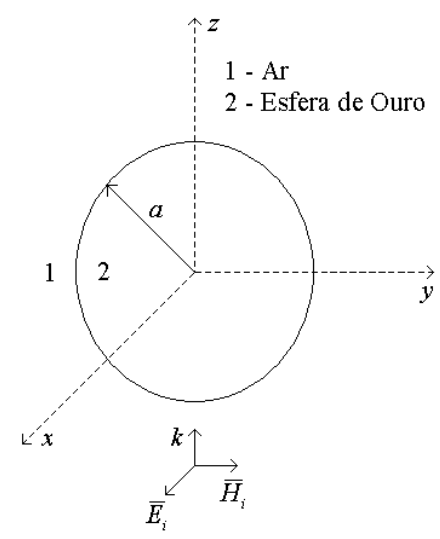

Fig. 1. Geometria do problema de espalhamento para o caso de uma esfera.

\section{A. Distribuição do campo próximo}

As Figs. 2-4 mostram exemplos da distribuição da intensidade do campo elétrico $\left|E_{x}\right|$ ao longo dos eixos $x, y$ e $z$, respectivamente. Estas curvas foram calculadas para $\lambda=550 \mathrm{~nm}, a=60 \mathrm{~nm}$ e $E_{i 0}=1 \mathrm{~V} / \mathrm{m}$ utilizando o modelo de Mie

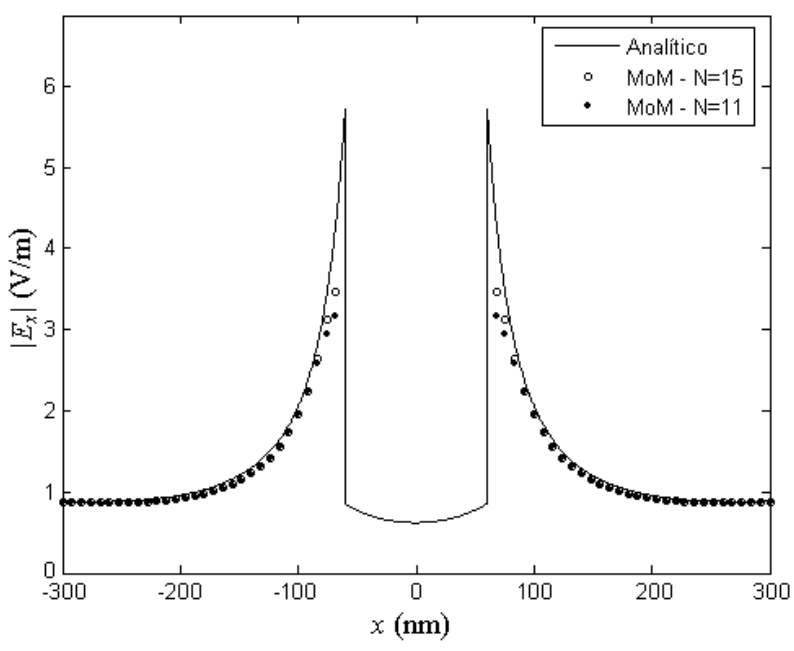

Fig. 2. Distribuição de $\left|E_{x}\right|$ ao longo de $x$ para $z=y=0$.

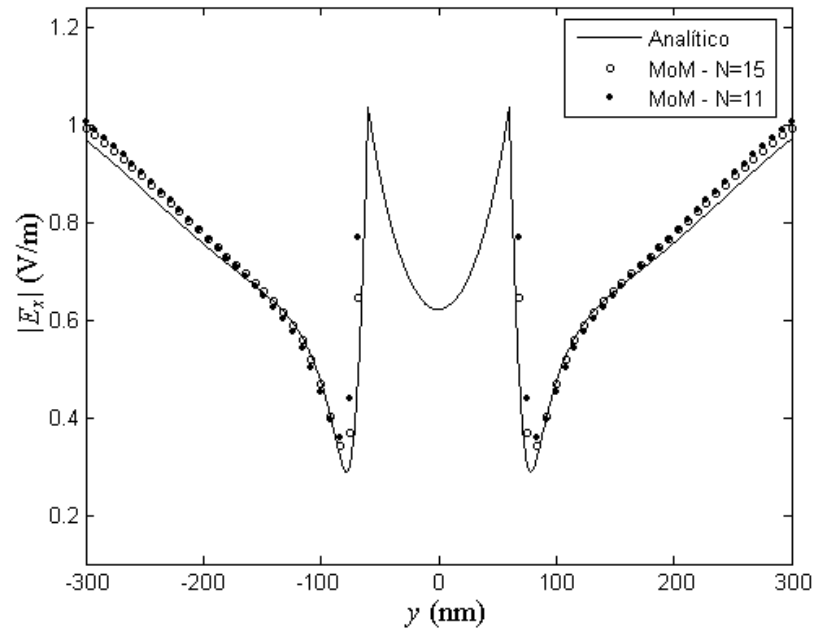

Fig. 3. Distribuição de $\left|E_{x}\right|$ ao longo de $y$ para $x=z=0$.

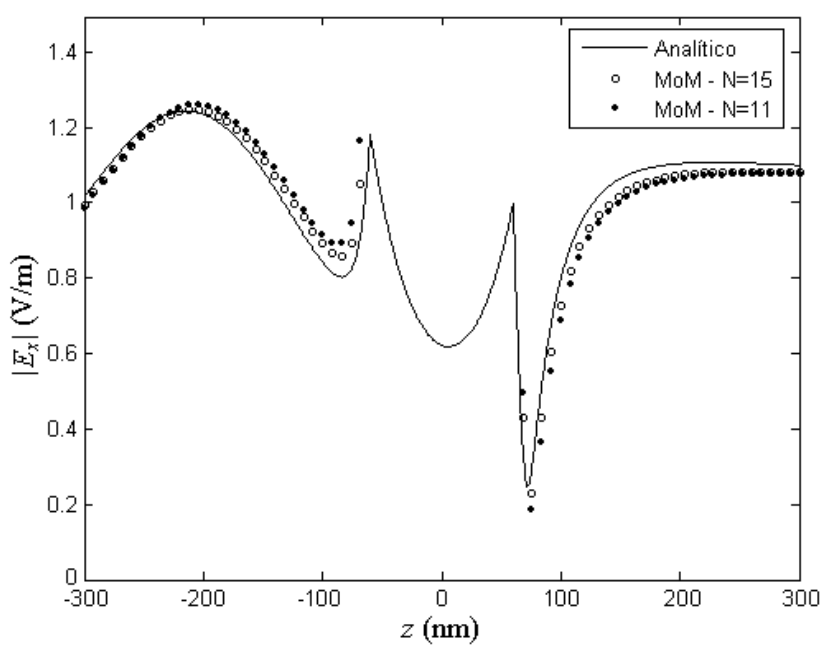

Fig. 4. Distribuição de $\left|E_{x}\right|$ ao longo de $z$ para $x=y=0$. 
e o MoM. Nos resultados obtidos pelo MoM foram utilizadas discretizações de $N=11$ e 15 ao longo da diagonal da esfera, ou seja, 11 e 15 divisões no diâmetro $2 a$ da esfera. Com estes valores obteve-se um total de 2217 e 5373 componentes de campo elétrico a serem determinadas pelo MoM no interior da esfera, para $N=11$ e 15 respectivamente. Observa-se uma boa convergência do método numérico com estes valores.

Os resultados destas figuras mostram apenas a componente $x$ do campo elétrico, pois verificou-se que as outras componentes são muito pequenas. Observa-se destes resultados que o campo elétrico é mais intenso nas regiões próximas das esferas ao longo do eixo $x$ (Fig. 2), isto é devido a polarização da onda plana incidente, pois com esta polarização o campo externo é normal à superfície da esfera nestes pontos, e com isto ocorre mais indução de carga elétrica.

Estes resultados foram calculados para $\lambda=550 \mathrm{~nm}$ e $a=60 \mathrm{~nm}$. A forma de distribuição do campo elétrico possui um comportamento semelhante para outros valores de $\lambda$ e $a$, mas a intensidade do campo possui variação com estes parâmetros, como é mostrado na próxima seção.

\section{B. Resposta espectral}

Depois da análise da distribuição do campo próximo da esfera, foi feita uma análise paramétrica do problema, onde os valores de $\lambda$ e $a$ foram modificados. O raio da esfera foi variado de $10 \mathrm{~nm}$ a $100 \mathrm{~nm}$, e o comprimento de onda de $500 \mathrm{~nm}$ a $1000 \mathrm{~nm}$. Os resultados obtidos são apresentados na Fig. 5, onde os campos foram calculados próximo da superfície da esfera no ponto $(1,09 a ; 0 ; 0)$. Estas curvas foram obtidas através do modelo analítico de Mie.

Os resultados desta figura mostram que o comprimento de onda de ressonância da esfera $\left(\lambda_{\text {res }}\right)$ aumenta para valores maiores de $a$, por exemplo, para $a=10 \mathrm{~nm}$ tem-se $\lambda_{\text {res }}=525 \mathrm{~nm}$, e para $a=60 \mathrm{~nm}$ tem-se $\lambda_{\text {res }}=550 \mathrm{~nm}$ (Fig. 5).
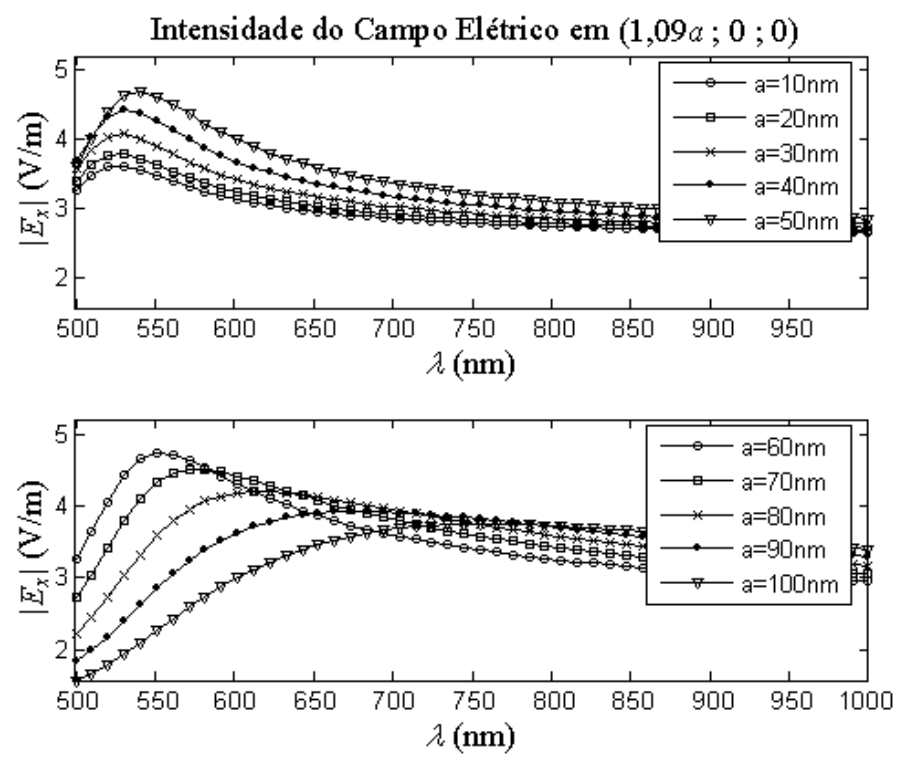

Fig. 5. Variação de $\left|E_{x}\right|$ no ponto $(1,02 a ; 0 ; 0)$ em função de $a$ e $\lambda$.

\section{RESUlTADOS PARA O CASO DE DUAS ESFERAS}

Nesta seção analisamos o problema de espalhamento ilustrado na Fig. 6, onde existem duas esferas de ouro de raios $a_{1}$ e $a_{2}$ centralizadas em $\left(a_{1}+d / 2 ; 0 ; 0\right)$ e $\left(-a_{2}-d / 2 ; 0 ; 0\right)$, respectivamente, sendo $d$ a distância entre elas. A onda plana incidente nesta estrutura possui a mesma forma utilizada para o caso anterior, a qual é dada por (2) e (3). Na análise deste problema utilizou-se também $E_{i 0}=1 \mathrm{~V} / \mathrm{m}$.

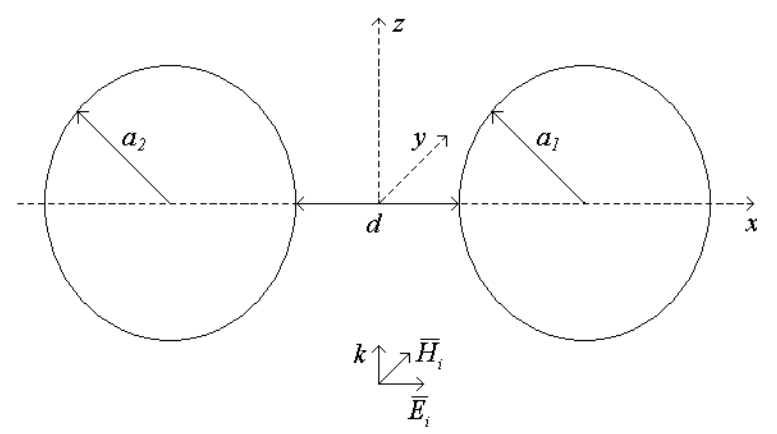

Fig. 6. Geometria do problema de espalhamento para o caso de duas esferas.

Para a análise numérica deste problema, vários códigos MoM foram desenvolvidos, e depois um conjunto de nove simulações foi realizado, variando-se os valores de $a_{1}, a_{2}$ e $d$. Utilizando a notação $\left(a_{1} ; a_{2} ; d\right)$ para cada simulação, o conjunto analisado foi $(60 ; 60 ; 120),(60 ; 60 ; 60),(60 ; 60$; $-9),(60 ; 40 ; 120),(60 ; 40 ; 60),(60 ; 40 ;-9),(60 ; 20 ; 120)$, $(60 ; 20 ; 60),(60 ; 20 ;-9)$, onde todas as dimensões são em nm. Valores $d<0$ são os casos onde as esferas se sobrepõem. Utilizou-se uma discretização de $N=11$ para cada esfera em todas as simulações, com este valor, obteve-se um sistema de ordem 4434 na aplicação do MoM. A seguir são apresentados os resultados destas simulações.

\section{A. Distribuição do campo próximo}

Para visualizar a variação do campo próximo das esferas analisadas, as Figs. 7-9 mostram a distribuição da intensidade do campo elétrico $\left(\left|E_{x}\right|\right)$ ao longo do eixo $x$, para todos os casos simulados, no comprimento de onda $\lambda=660 \mathrm{~nm}$.

Nestas figuras os campos foram calculados no eixo $x$, pois nesta direção o campo é mais intenso, devido ao mesmo motivo da polarização da onda incidente observado para o caso de uma esfera. Estes resultados mostram que a medida que aproximamos as esferas obtemos campos mais intensos nas superfícies das esferas na direção $x$, sendo que entre as esferas o campo é maior. Isto é devido ao efeito eletrostático de atração de cargas elétricas de sinais opostos na região entre as esferas.

Quando se utilizam esferas de diferentes tamanhos, o campo elétrico tende a ficar mais intenso próximo da menor esfera. Foi observado que este efeito é mais acentuado quando as esferas se aproximam para o caso com menor valor de $a_{2}$ mostrado na Fig. 9.

O caso que obteve campo mais intenso é o com $a_{1}=60 \mathrm{~nm}$, $a_{2}=20 \mathrm{~nm}$ e $d=-9 \mathrm{~nm}$ mostrado na Fig. 9. Este resultado também pode ser explicado pelo efeito eletrostático de 

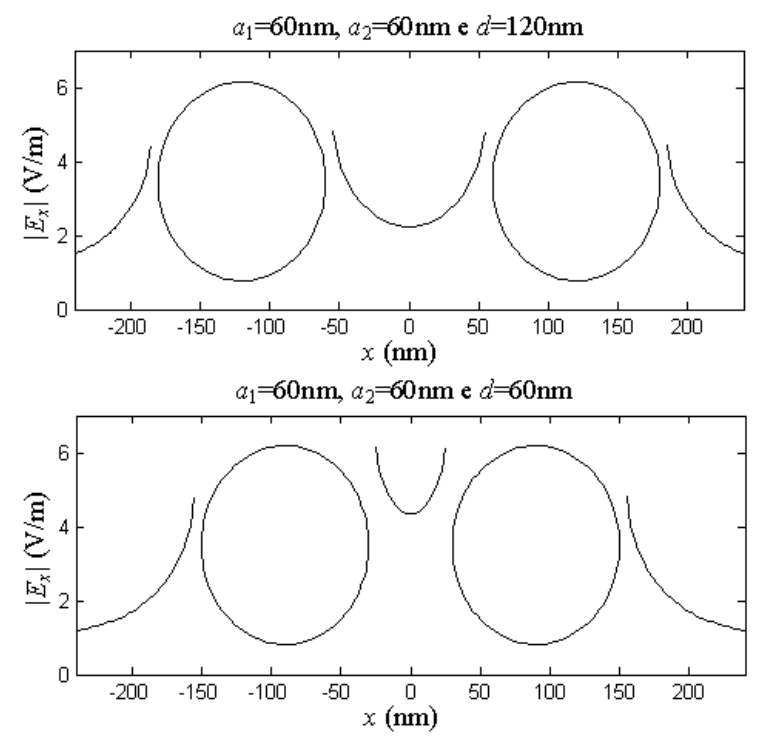

$a_{1}=60 \mathrm{~nm}, a_{2}=60 \mathrm{~nm}$ e $d=9 \mathrm{~nm}$

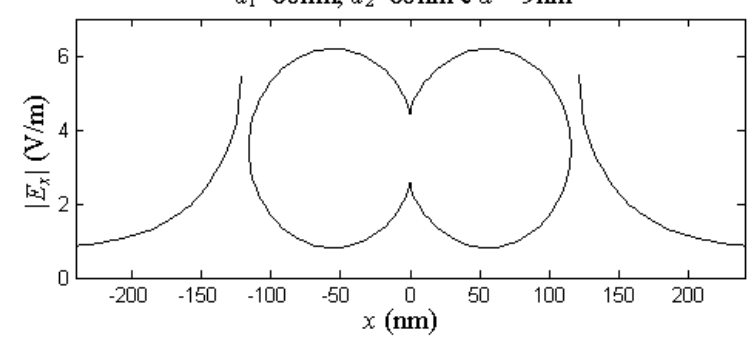

Fig. 7. Variação de $\left|E_{x}\right|$ ao longo de $x$ para os casos $a_{1}=60 \mathrm{~nm}$ e $a_{2}=60 \mathrm{~nm}$.
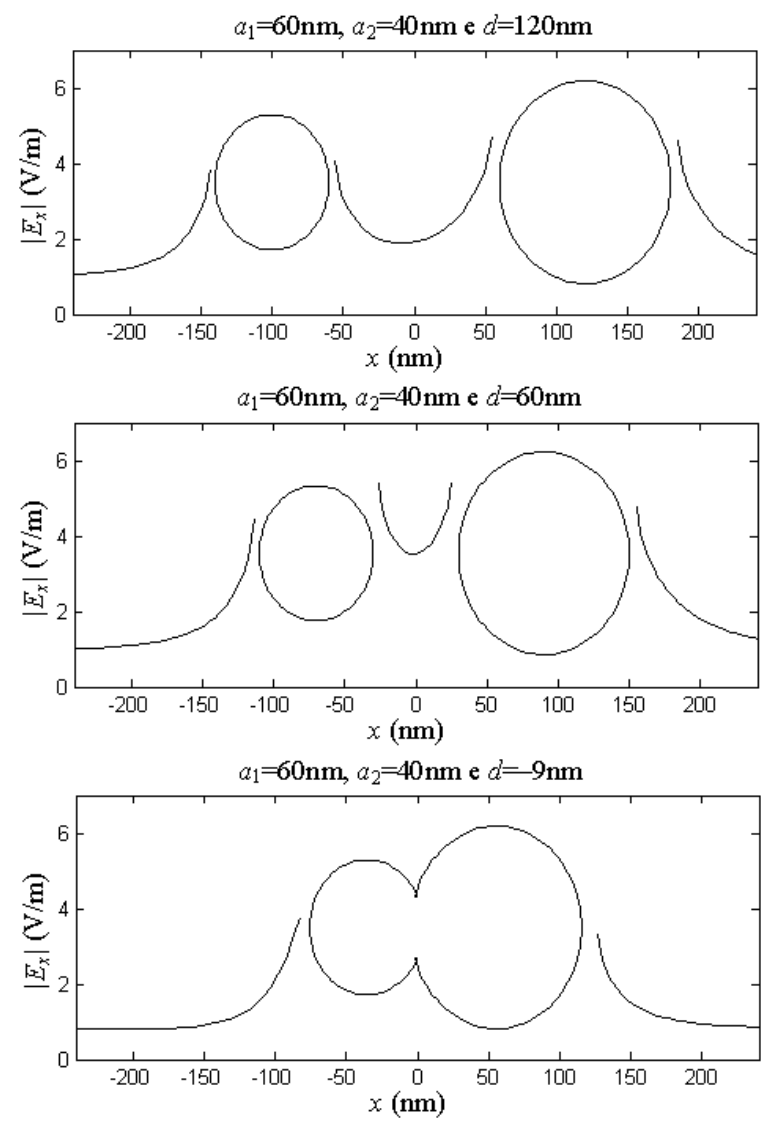

Fig. 8. Variação de $\left|E_{x}\right|$ ao longo de $x$ para os casos $a_{1}=60 \mathrm{~nm}$ e $a_{2}=40 \mathrm{~nm}$.
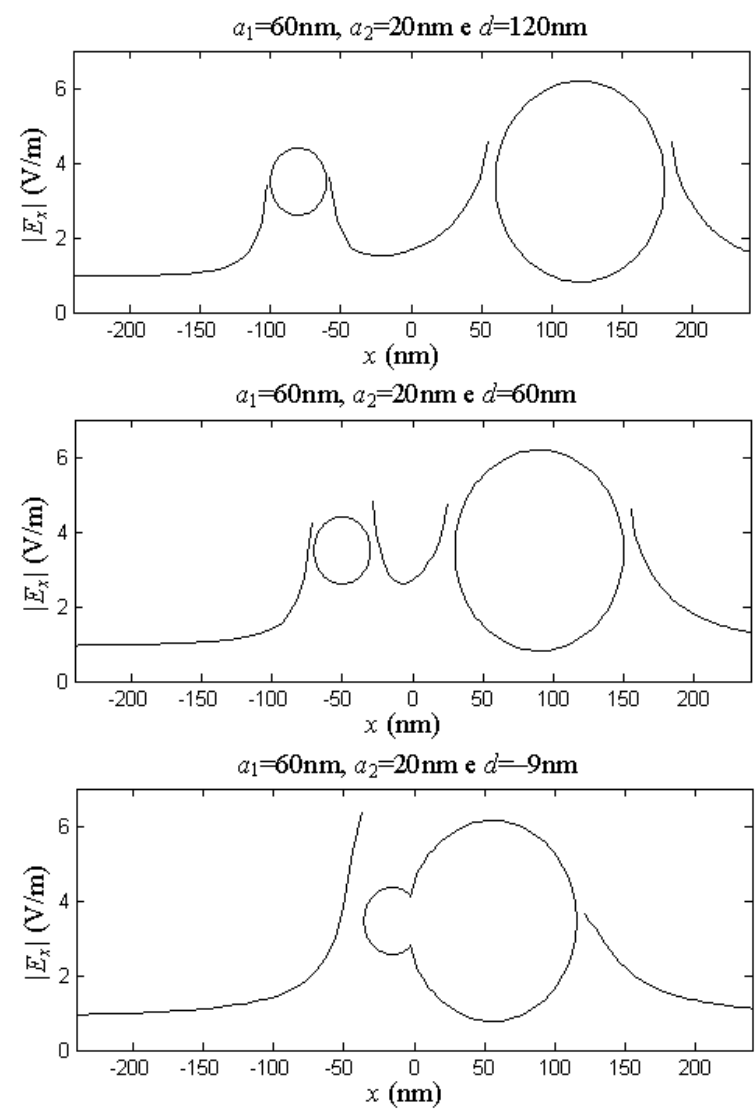

Fig. 9. Variação de $\left|E_{x}\right|$ ao longo de $x$ para os casos $a_{1}=60 \mathrm{~nm}$ e $a_{2}=20 \mathrm{~nm}$

distribuição de cargas elétricas em condutores, onde as cargas tendem a se acumular nas pontas do condutor. Este efeito é conhecido na eletrostática como o "poder das pontas". Este caso da Fig. 9 sugere utilizar uma estrutura simétrica com quatro esferas, sendo as duas esferas menores de frente uma para outra, formando assim uma estrutura parecida com nanoantenas bowtie [10]-[11] e cônicas [12]. Com isto podese obter campos mais intensos no centro da estrutura para aplicações em amplificação do campo óptico próximo.

Os resultados obtidos nesta seção foram calculados para $\lambda=660 \mathrm{~nm}$, mas foram feitos cálculos para outros comprimentos de onda de 500nm a 1000nm. Observou-se que a forma de distribuição do campo elétrico para outros valores de $\lambda$ possui um comportamento semelhante daqueles apresentados nas Figs. 7-9, mas a intensidade do campo possui variação com $\lambda$, como ocorreu para o caso de uma esfera (Fig. 5). Na próxima seção é apresentada a resposta espectral para o caso de duas esferas.

Para observar melhor a diferença entre os casos $d=60 \mathrm{~nm}$ e $d=-9 \mathrm{~nm}$ da Fig. 9, as Figs. 10-11 mostram, respectivamente, a distribuição da intensidade do campo elétrico no plano $x y$ destes casos. Nestes gráficos é observado, novamente, que os campos próximos da menor esfera para o caso $d=-9 \mathrm{~nm}$ são mais intensos que os do caso $d=60 \mathrm{~nm}$ (observe a diferença nas escalas destes dois gráficos). Nestes gráficos observa-se também o tamanho da região de confinamento onde o campo é mais intenso, para o caso $d=-9 \mathrm{~nm}$ (Fig. 11) esta região é menor que a do caso $d=60 \mathrm{~nm}$ (Fig. 10). 


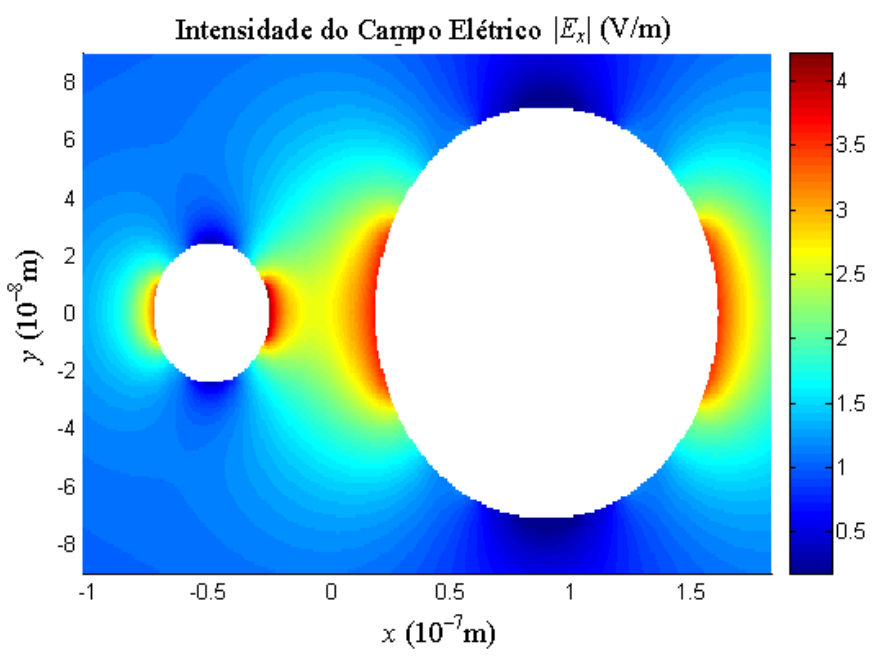

Fig. 10. Distribuição de $\left|E_{x}\right|$ no plano $x y$ para $a_{1}=60 \mathrm{~nm}, a_{2}=20 \mathrm{~nm}$ e $d=60 \mathrm{~nm}$.

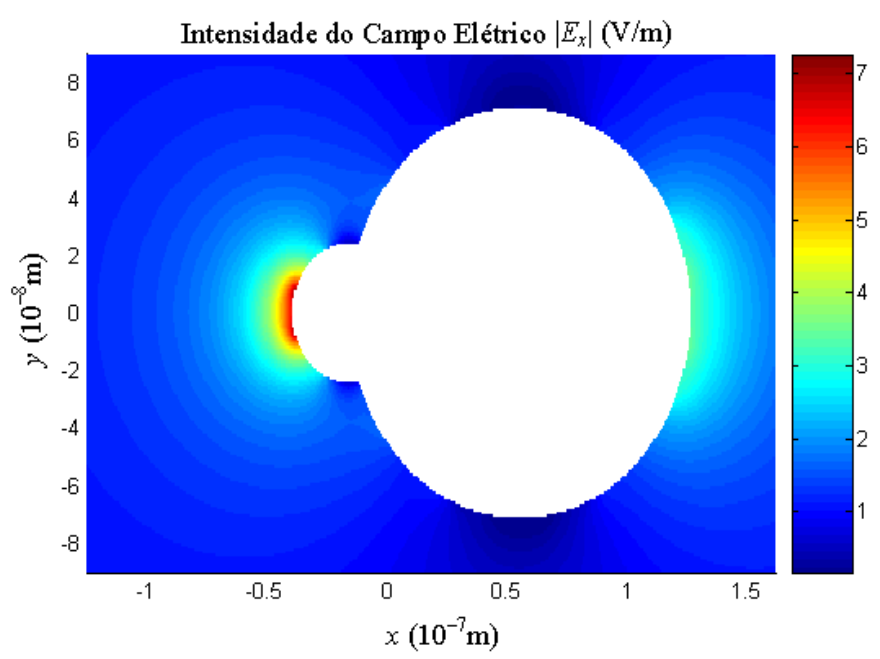

Fig. 11. Distribuição de $\left|E_{x}\right|$ no plano $x y$ para $a_{1}=60 \mathrm{~nm}, a_{2}=20 \mathrm{~nm}$ e $d=-9 \mathrm{~nm}$.

\section{B. Resposta espectral}

As Figs. 12-14 mostram a variação da intensidade do campo elétrico, em alguns pontos, em função do comprimento de onda. Os campos nestas figuras foram calculados próximos das superfícies das esferas nos pontos $\mathrm{P} 1\left(-2,09 a_{2}-0,5 d ; 0 ; 0\right)$, $\mathrm{P} 2\left(0,09 a_{2}-0,5 d ; 0 ; 0\right)$ e P3 $\left(2,09 a_{1}+0,5 d ; 0 ; 0\right)$. Estes pontos estão à mesma distância da esfera que aqueles calculados para o caso de uma esfera (Fig. 5). Os pontos P1 e P2 estão ao redor da esfera de raio $a_{2}$ e o ponto $\mathrm{P} 3$ próximo da esfera de raio $a_{1}$.

As curvas mostradas nestas figuras apresentam pequenas variações, que são diferentes daquelas da Fig. 5. Isto é devido as aproximações do método numérico e da discretização utilizada. Mas se for considerada a envoltória destas curvas, observa-se que cada uma apresenta um ponto de máximo diferente, sendo que nestes pontos ocorre aproximadamente a ressonância, por exemplo, $\lambda_{\text {res }} \approx 750 \mathrm{~nm}$ na Fig. 12 ( $\left.d=-9 \mathrm{~nm}\right)$.

Comparando as ressonâncias das estruturas com duas esferas (Figs. 12-14) com aquela com uma esfera e raio $a=60 \mathrm{~nm}$ (Fig. 5), observa-se que no primeiro caso os valores
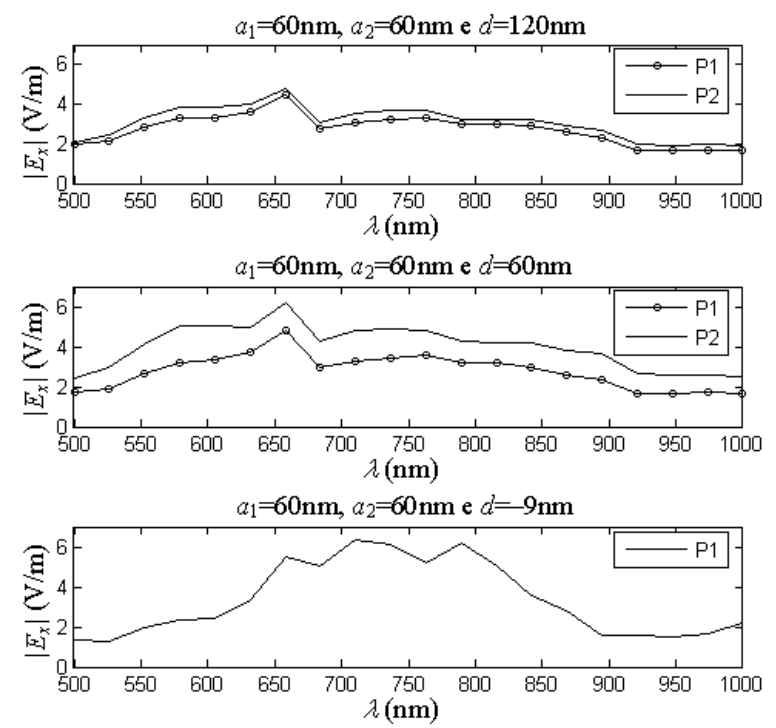

Fig. 12. $\left|E_{x}\right|$ versus $\lambda$ nos pontos P1, P2 e P3 para o caso $a_{1}=60 \mathrm{~nm}$ e $a_{2}=60 \mathrm{~nm}$.
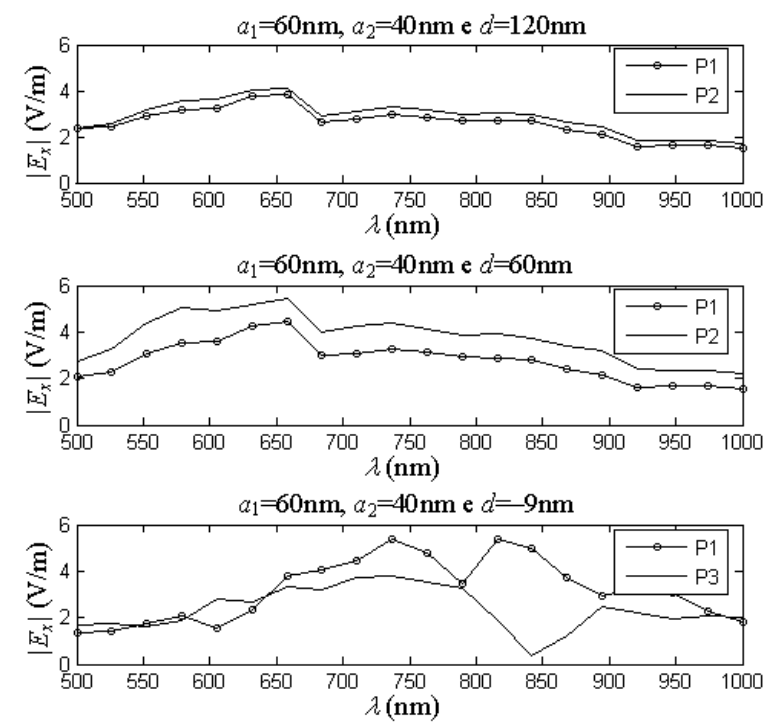

Fig. 13. $\left|E_{x}\right|$ versus $\lambda$ nos pontos P1, P2 e P3 para o caso $a_{1}=60 \mathrm{~nm}$ e $a_{2}=40 \mathrm{~nm}$.
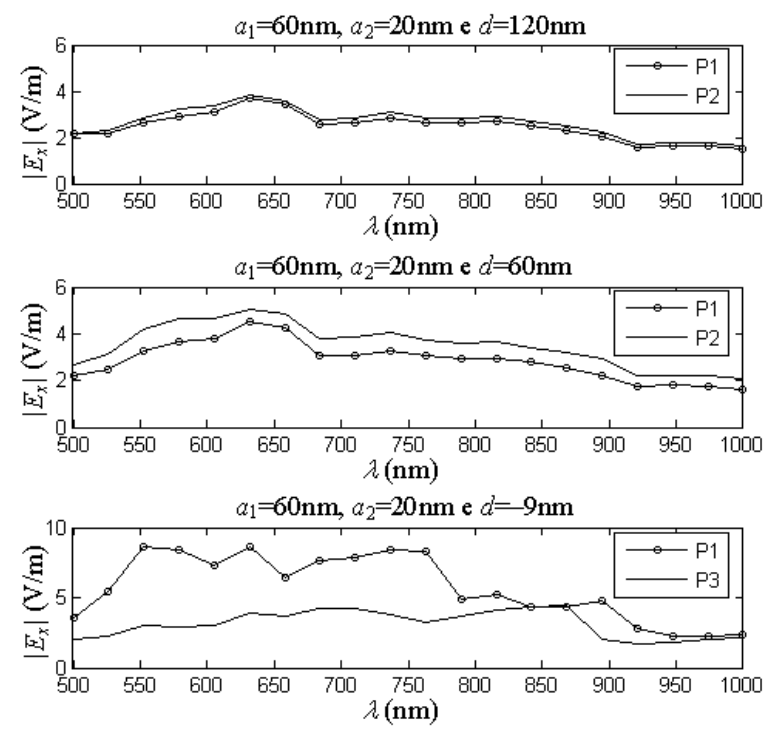

Fig. 14. $\left|E_{x}\right|$ versus $\lambda$ nos pontos P1, P2 e P3 para o caso $a_{1}=60 \mathrm{~nm}$ e $a_{2}=20 \mathrm{~nm}$. 
de $\lambda_{\text {res }}$ são maiores que aquele do segundo caso. Isto mostra que nanoantenas maiores possuem ressonâncias em maiores comprimentos de onda. Outra comparação entre estes dois casos é com relação à intensidade do campo elétrico próximo das esferas. Os casos com duas esferas apresentam valores maiores que aqueles obtidos com uma esfera. Por exemplo, o valor máximo obtido para uma esfera é aproximadamente $\left|E_{x}\right|=4,8 \mathrm{~V} / \mathrm{m}$ para o caso com $a=60 \mathrm{~nm}$ em $\lambda_{\text {res }}=550 \mathrm{~nm}$ (Fig. 5 ). Para os casos com duas esferas analisado o valor máximo é próximo de $\left|E_{x}\right|=9 \mathrm{~V} / \mathrm{m}$ em $\lambda_{\text {res }}=550 \mathrm{~nm}$ e $d=-9 \mathrm{~nm}$ (Fig. 14).

Analisando agora apenas os casos com duas esferas mostrados nas Figs. 12-14, primeiro se observa que ao aproximar as esferas, os valores de $\lambda_{\text {res }}$ diminuem, pois o tamanho total da antena diminui. Mas quando $d<0$, que são os casos onde as esferas se sobrepõem, o valor de $\lambda_{\text {res }}$ aumenta.

\section{CONCLUSÕES}

Neste trabalho foi apresentada uma análise teórica do campo próximo de nanoantenas formadas por partículas esféricas de ouro. De todos os casos analisados, a nanoantena que apresentou melhores resultados, em termos de confinamento e amplificação do campo próximo, é aquela mostrada na Fig. 11, onde as esferas são sobrepostas. Para melhorar as características desta antena, os resultados apresentados sugerem a análise de uma forma simétrica desta antena com quatro esferas, sendo as esferas menores posicionadas próximas. Este problema será analisado em trabalhos futuros.

\section{REFERÊNCIAS}

[1] M. L. Brongersma, and P. G. Kik, Surface Plasmon Nanophotonics, Netherlands: Springr, 2007.

[2] L. Novotny, and B. Hecht, Principles of Nano-Optics, New York: Cambridge, 2006

[3] H. A. Atwater, "As surpreendentes promessas da plamônica", Scientific American Brasil, pp. 58-65, 2007.

[4] D. W. Pohl, "Near field optics as an antenna problem"', Near Field: Principles and Applications, The second Asia-Pacific Workshop on Near Field Optics, Beijing, China October 20-23, pp. 9-21, 1999.

[5] J. Li, and N. Engheta, "Core-shell nanowire optical antenna fed by slab waveguides", IEEE Trans. Ant. Propag., vol. 55, N11, pp. 3018-3026, November 2007.

[6] B. Hecht, et. al., "Prospects of resonant optical antennas for nanoanalysis", Chimia, vol. 60, N11, pp. 765-769, 2006.

[7] O. L. Nuskens, V. Giannini, J. A. Sánches-Gil, and J. G. Rivas, "Optical scattering resonances of single and coupled dimer plasmonic nanoantennas", Optic Express, vol. 15, N26, pp.17736-17746, December 2007.

[8] E. Cubukcu, et. al., "Plasmonic laser antenna", Applied Physics Letters, vol. 89,2006

[9] T. H. Taminiau, et. al., "Near-fields driving of a optical monopole antenna"', J. Opt. A: Pure Appl. Opt., vol. 9, pp. S315-S321, 2007.

[10] A. Sundaramurthy, et.al., "Toward nanometer-scale optical photolithography: utilizing the near-field of bowtie optical nanoantennas", Nano Letters, vol. 6, N3, pp. 355-360, November 2006.

[11] P. Mühlschlegel, U.-J. eisler, O. J. F. Martin, B. Hecht, D. W. Pohl, "Resonant optical antennas", Science, vol. 308, N5728, pp. 1607-1609, June 2005.

[12] R. Kappeler, et. al., "Field computation of optical antennas", J. Comput. Theor. Nanosci., vol. 4, N3, pp. 686-691, 2007.

[13] W. Rechberger, et. al., "Optical properties of two interacting nanoparticles", Optics Communications, vol. 220, pp.137-141, 2003.

[14] J. A. Stratton, Electromagnetic Theory, New York: McGraw-Hill, 1941.
[15] D. E. Livesay, and K. M. Chen, "Electromagnetic fields induced inside arbitrary shaped biological bodies", IEEE Trans. Micro. Theo. Thec., vol. 22, N12, pp. 1273-1280, December 1974. 